\title{
How to Assess the Sustainable Growth of Non-listed SMEs: Based on Factor Analysis Model \\ Jingxian Liu ${ }^{\mathrm{ab} *}$, Yingyu $\mathrm{Wu}^{a}$ \\ ${ }^{a}$ School of Economics and Management, Southeast University, China \\ ${ }^{\mathrm{b}}$ School of Business, Nantong University, China \\ *Corresponding author: Jingxian Liu, PhD,380369224@qq.com
}

\begin{abstract}
Factor analysis model is utilized to find out the key determinants of sustainable development of nonlisted companies in the manufacture of communication equipment industry.21 determinants from 4 dimensions in the performance comprehensive assessment system are chosen and 7 determinants are put forward as Principal Component indicators in the result of Principal Component Analysis. The result shows profitability management, efficiency of using assets and sales capacity play pivotal role to unlisted SMEs.
\end{abstract}

Key words: sustainable growth rate; factor analysis ;communication-equipment manufacturing; non-listed company; operation performance ;empirical analysis; SMEs

\section{Introduction}

Sustainable growth is treated as the main aim of management which enterprises are supposed to pursue, of which the definition can date back as far as the formal work of Babcok $^{1}(1970)$.The definition of sustainable growth is recognized as maximum rate at which a company sales can increase with operating and financial constraints by Higgins ${ }^{2}$ (1977).Pioneering in many researchers' efforts, focused at the large listed companies, Gerard and Michael $^{3}$ (2005) utilize the sustainable growth for evaluating the long run performance of bank mergers. The most famous SGR models based on the accounting rationale are created by Higgins $^{4}(2000)$ and Van Home ${ }^{5}(1988)$.In an extension to the traditional models, Lihua Wang etal. $^{6}$ (2015) acclimatize the model in China. As a multifaceted metric, a growing body of empirical literature have demonstrated the significance to identify the drives of enterprises' sustainable growth.

To provide a growth impetus for sustainable economic development in China, structural reform of the supply front is advocated. In 2015,the added value calculated in communication equipment manufacturing industry being part of high and new technology has recorded a $12.7 \%$ growth compared to the one in $2014^{7}$.Under the circumstance of promotion of "Internet plus initiative” programme, the industry gains a promising expectation. This study attempts to provide a way into the observation of non-listed small and medium enterprises (SMEs)in communication equipment manufacturing industry for research on enterprises' operations and development.

\section{Study Design}

\subsection{Sample and performance assessment system}

394 enterprises are chosen as a sample and all the data are from the Oriana Asia-Pacific 
Enterprises database. To identify active SMEs matched with CSIC39-Computer, communication and other electronic equipment in China, of which the latest year 2014 of accounts can be possessed, it provides the authors with an initial set of 395 companies. Only companies in the normal business operation are selected, the others active but involved in the rescue plan, default of payment, insolvency proceedings, etc. are deleted. Totally 394 companies are left to screen out the number of non-listed companies, which are all unlisted companies. The operation performance comprehensive assessment system generally includes three sections profitability, operating capacity, and solvency at least. In this paper, the productivity analysis into the assessment system is considered either. Financial indices are classified into Table 1.

Table 1 -Financial indices classification

\begin{tabular}{|l|l|l|l|l|l|}
\hline \multicolumn{2}{|c|}{ Profitability Analysis } & & Operational Capacity Analysis & & Solvency Analysis \\
\hline X1 & ROE using P/L before tax & X8 & Net assets turnover & X15 & Solvency ratio (Liability based) \\
\hline X2 & ROA using P/L before tax & X9 & Stock turnover & X16 & Gearing \\
\hline X3 & ROE using Net income & X10 & Collection period & & Productivity Analysis \\
\hline X4 & ROA using Net income & X11 & Credit period & X17 & Profit per employee \\
\hline X5 & Profit Margin & & Solvency Analysis & X18 & Operating revenue per employee \\
\hline X6 & Gross Margin & X12 & Current ratio & X19 & Shareholders funds per employee \\
\hline X7 & EBIT Margin (\%) & X13 & Liquidity ratio (x) & X20 & Working capital per employee \\
\hline & & X14 & Solvency ratio (Asset based) & X21 & Total assets per employee \\
\hline
\end{tabular}

Note: ROE—return on equity, ROA — return on asset

\subsection{Test of suitability of standardized data}

To eliminate parameters' incommensurability due to the difference of dimension and unit or positive and negative, all the collection of data should be standardized. The mean values of 21 indicators, subtracted by each indicator, divide the standard deviation. The standardized mean value equals 0 and the standard deviation equals 1 .

To investigate the existence of the linear dependence before the application of factor analysis, KMO measure and Bartlett's Test of Sphericity are conducted to test correlation. In the result of Table2, KMO's value 0.766 means that linear correlation between the variables does not appear to be much different, meanwhile the significance of Bartlett's Test of Sphericity obviously is less than 0.05 ,the rejection of Spherical assumption states the variances are not respectively independent. Analyses above manifest the indicators chosen are suitable for factor analysis.

Table 2-Kaiser-Meyer-Olkin Measure and Bartlett's Test of Sphericity

\begin{tabular}{|c|c|c|}
\hline \multicolumn{2}{|c|}{ Kaiser-Meyer-Olkin Measure of Sampling Adequacy } & 766 \\
\hline Bartlett's Test of Sphericity & Approx. Chi-Square & 4240.875 \\
\hline \multicolumn{2}{|c|}{ Df. } & 210 \\
\hline Sig. & .000 \\
\hline
\end{tabular}

\section{Factor analysis model}

\subsection{Principal Component Analysis}

Principal Component Analysis is applied to calculate initial common factor Eigenvalue, 
contribution and cumulative contribution of variable of 21 indicators. Seen from Table 3, the communalities of variables are 1 , which implies each variable can be explained. Wherein, ROE using P/L before tax, ROE using Net income, Profit Margin, Current ratio, Liquidity ratio, Solvency ratio (Asset based) and Solvency ratio (Liability based)can be extracted as common factors, because the 7 variables' eigenvalues are more than 0.9.ScreePlot comes into use to consider the amount of common factors, and from the Fig. 1, the drawing line gradually tends to smooth since the $7^{\text {th }}$ factor. Therefore, the 7 common factors are reasonable. In Table 4 , the result of the accumulated variance achieves 80.593 percent of total variance, when 7 common factors are extracted. As well as the eigenvalues above, the variance signifies 7 common factors can explain original information adequately which possess representative. The total score of SMEs' sustainable growth is:

$$
F_{i}=a_{i 1} \times x_{1}+a_{i 2} \times x_{2}+\ldots \ldots+a_{i j} \times x_{j}(i=1, \ldots \ldots, 7 ; j=1, \ldots \ldots, 21)
$$

Note: F-total score of comprehensive evaluation to SMEs' sustainable growth

a-contribution rate of variance

$\mathrm{x}$ - score of each factor

Table 3 - Communalities

\begin{tabular}{|l|r|r|l|r|r|l|r|r|}
\hline & Initial & Extraction & & Initial & Extraction & & Initial & Extraction \\
\hline $\mathrm{x} 1$ & 1.000 & .923 & $\mathrm{x} 8$ & 1.000 & .878 & $\mathrm{x} 15$ & 1.000 & .912 \\
\hline $\mathrm{x} 2$ & 1.000 & .838 & $\mathrm{x} 9$ & 1.000 & .324 & $\mathrm{x} 16$ & 1.000 & .732 \\
\hline $\mathrm{x} 3$ & 1.000 & .939 & $\mathrm{x} 10$ & 1.000 & .762 & $\mathrm{x} 17$ & 1.000 & .739 \\
\hline $\mathrm{x} 4$ & 1.000 & .786 & $\mathrm{x} 11$ & 1.000 & .814 & $\mathrm{x} 18$ & 1.000 & .807 \\
\hline $\mathrm{x} 5$ & 1.000 & .901 & $\mathrm{x} 12$ & 1.000 & .955 & $\mathrm{x} 19$ & 1.000 & .860 \\
\hline $\mathrm{x} 6$ & 1.000 & .552 & $\mathrm{x} 13$ & 1.000 & .922 & $\mathrm{x} 20$ & 1.000 & .627 \\
\hline $\mathrm{x} 7$ & 1.000 & .856 & $\mathrm{x} 14$ & 1.000 & .931 & $\mathrm{x} 21$ & 1.000 & .867 \\
\hline
\end{tabular}

Note: Extraction Method: Principal Component Analysis

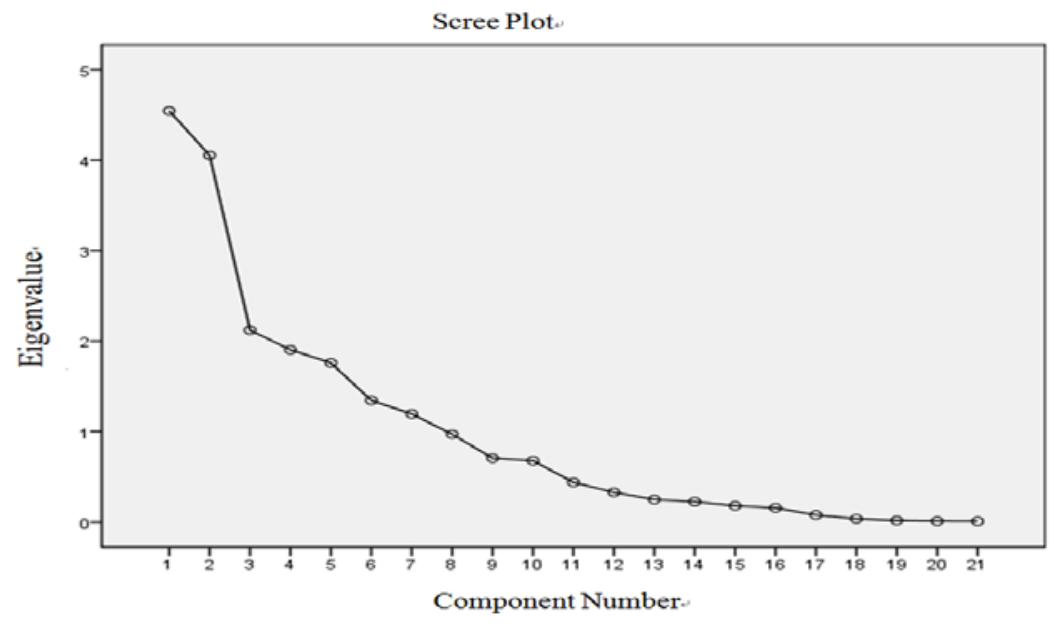

Fig. 1 - ScreePlot

\subsection{Economic interpretation of factor analysis}

Through the varimax orthogonal rotation, factor loading matrix (Table 5) and Component Score Coefficient Matrix (Table 6) display the following information: Component 1 mainly includes $\mathrm{X}_{2}, \mathrm{X}_{4}, \mathrm{X}_{5}, \mathrm{X}_{7}, \mathrm{X}_{17}$, which can be on behalf of profitable growth; Component2 mainly includes $\mathrm{X} 14, \mathrm{X} 15, \mathrm{X} 19, \mathrm{X} 20, \mathrm{X} 21$, which represents efficiency of using assets;Component3 
mainly includes X1,X3,X8,which means sales ability; Component4 mainly includes $\mathrm{X} 10, \mathrm{X} 11$,which shows operating capacity; Component5 mainly includes $\mathrm{x} 18$, which implies labour productivity; Component 6 mainly includes X12,X13, which means cashability; Component 7 mainly includes X6,X9,X16, which shows financing capacity. Component Score Covariance Matrix (Table 7) validates the rationality of extracting principal component. Finally, the total score to evaluate the sustainable growth capacity is in the following:

$F=\sum_{\mathrm{i}=1}^{7}\left(V_{i} / V^{T}\right) \mathrm{F}_{\mathrm{i}}=\left(19.434 \mathrm{~F}_{1}+12.931 F_{2}+12.452 F_{3}+11.386 F_{4}+9.580 F_{5}+8.934 F_{6}+5.877 F_{7}\right) / 80.593$

Table 4 - Total Variance Explained

\begin{tabular}{|c|c|c|c|c|c|c|c|c|c|}
\hline \multirow[b]{2}{*}{ Component } & \multicolumn{3}{|c|}{ Initial Eigenvalue } & \multicolumn{3}{|c|}{$\begin{array}{c}\text { Extraction Sums of Squared } \\
\text { loadings }\end{array}$} & \multicolumn{3}{|c|}{$\begin{array}{c}\text { Rotation Sums of Squared } \\
\text { Loadings }\end{array}$} \\
\hline & Total & $\begin{array}{c}\% \text { of } \\
\text { Variance }\end{array}$ & $\begin{array}{c}\text { Cumulati } \\
\text { ve } \%\end{array}$ & & Total & $\begin{array}{c}\% \text { of } \\
\text { Variance }\end{array}$ & $\begin{array}{c}\text { Cumulative } \\
\%\end{array}$ & & Total \\
\hline 1 & 4.547 & 21.651 & 21.651 & 4.547 & 21.651 & 21.651 & 4.081 & 19.434 & 19.434 \\
\hline 2 & 4.054 & 19.302 & 40.954 & 4.054 & 19.302 & 40.954 & 2.715 & 12.931 & 32.364 \\
\hline 3 & 2.121 & 10.099 & 51.053 & 2.121 & 10.099 & 51.053 & 2.615 & 12.452 & 44.816 \\
\hline 4 & 1.904 & 9.067 & 60.120 & 1.904 & 9.067 & 60.120 & 2.391 & 11.386 & 56.202 \\
\hline 5 & 1.758 & 8.373 & 68.493 & 1.758 & 8.373 & 68.493 & 2.012 & 9.580 & 65.782 \\
\hline 6 & 1.345 & 6.406 & 74.898 & 1.345 & 6.406 & 74.898 & 1.876 & 8.934 & 74.716 \\
\hline 7 & 1.196 & 5.695 & 80.593 & 1.196 & 5.695 & 80.593 & 1.234 & 5.877 & 80.593 \\
\hline 8 & .970 & 4.618 & 85.211 & & & & & & \\
\hline 9 & .705 & 3.358 & 88.569 & & & & & & \\
\hline 10 & .678 & 3.231 & 91.799 & & & & & & \\
\hline 11 & .439 & 2.088 & 93.888 & & & & & & \\
\hline 12 & .328 & 1.562 & 95.450 & & & & & & \\
\hline 13 & .248 & 1.182 & 96.632 & & & & & & \\
\hline 14 & .224 & 1.067 & 97.699 & & & & & & \\
\hline 15 & .179 & .852 & 98.551 & & & & & & \\
\hline 16 & .157 & .746 & 99.296 & & & & & & \\
\hline 17 & .077 & .367 & 99.663 & & & & & & \\
\hline 18 & .035 & .167 & 99.830 & & & & & & \\
\hline 19 & .017 & .080 & 99.910 & & & & & & \\
\hline 20 & .010 & .050 & 99.959 & & & & & & \\
\hline 21 & .009 & .041 & 100.000 & & & & & & \\
\hline
\end{tabular}

Note: Extraction Method: Principal Component Analysis

\section{Conclusion}

In this paper, the authors select 21 drives to evaluate the sustainable growth capacity of unlisted SMEs and get the result that profitability management is the most important impact factor. Besides, efficiency of using assets and sales capacity are equally important. Moreover, SMEs still cannot ignore the importance of financing capacity and labour productivity. Although the study is influenced by the limitation of data collection and the evaluation 
indicators chosen, factor analysis is conducive to knowing important components of sustainable growth of SMEs objectively and comprehensively.

Table 5 - Rotated Component Matrix

\begin{tabular}{|c|c|c|c|c|c|c|c|}
\hline & \multicolumn{7}{|c|}{ Component } \\
\hline & 1 & 2 & 3 & 4 & 5 & 6 & 7 \\
\hline $\mathrm{x} 1$ & .604 & -.267 & .634 & -.191 & .192 & .063 & .090 \\
\hline $\mathrm{x} 2$ & .873 & .073 & -.167 & .018 & -.104 & .131 & .118 \\
\hline x3 & .563 & -.239 & .672 & -.217 & .229 & .119 & .001 \\
\hline $\mathrm{x} 4$ & .836 & .137 & -.124 & .008 & -.074 & .211 & .054 \\
\hline x5 & .866 & .082 & -.252 & .231 & -.038 & .013 & -.162 \\
\hline $\mathrm{x} 6$ & -.050 & .346 & .102 & -.027 & .278 & .276 & .515 \\
\hline $\mathrm{x} 7$ & .836 & .069 & -.239 & .255 & -.079 & .079 & -.136 \\
\hline $\mathrm{x} 8$ & .290 & -.517 & .678 & -.151 & .129 & -.131 & -.099 \\
\hline $\mathrm{x} 9$ & .130 & -.036 & -.111 & -.128 & -.185 & .166 & .464 \\
\hline $\mathrm{x} 10$ & -.131 & .250 & .155 & .725 & .292 & .213 & .045 \\
\hline x11 & -.228 & -.183 & .108 & .638 & .443 & .271 & -.200 \\
\hline $\mathrm{x} 12$ & .186 & -.570 & -.017 & -.118 & .447 & .612 & -.079 \\
\hline $\mathrm{x} 13$ & .222 & -.509 & -.057 & -.020 & .486 & .610 & -.039 \\
\hline x14 & -.054 & .784 & -.051 & -.426 & .112 & .337 & -.062 \\
\hline x15 & -.072 & .774 & -.002 & -.433 & .119 & .318 & -.072 \\
\hline x16 & .058 & .058 & .046 & .260 & .008 & -.310 & .748 \\
\hline x17 & .798 & .091 & -.263 & .130 & .077 & -.035 & -.013 \\
\hline x18 & 193 & .310 & .340 & .008 & -.711 & -.207 & -.104 \\
\hline x19 & -.081 & .832 & .327 & .074 & -.042 & .204 & -.064 \\
\hline x20 & -.056 & .543 & .267 & .196 & -.424 & -.196 & .024 \\
\hline x21 & -.063 & 591 & .463 & .466 & -.283 & .015 & -.040 \\
\hline
\end{tabular}

Note: Extraction Method: Principal Component Analysis. Rotation Method : Varimax with Kaiser Normalization. A Rotation converged in 6 iterations.

Table 6 - Rotated component Matrix

\begin{tabular}{|l|r|r|r|r|r|r|r|}
\hline & \multicolumn{7}{|c|}{ Component } \\
\cline { 2 - 8 } & 1 & 2 & 3 & 4 & 5 & 6 & 7 \\
\hline x1 & -.010 & .052 & .364 & -.005 & .002 & .009 & .074 \\
\hline x2 & .216 & .037 & -.005 & -.010 & -.078 & -.036 & .104 \\
\hline x4 & -.017 & .093 & .383 & -.015 & -.009 & .033 & -.001 \\
\hline x5 & .213 & .089 & .011 & -.014 & -.101 & -.002 & .052 \\
\hline x6 & .247 & -.049 & -.063 & .014 & .020 & .054 & -.115 \\
\hline x7 & -.027 & .203 & .065 & -.107 & -.060 & .108 & .436 \\
\hline x8 & -.250 & -.032 & -.066 & .023 & -.032 & .069 & -.095 \\
\hline x9 & .032 & -.079 & .350 & .040 & .062 & -.015 & -.089 \\
\hline x10 & .041 & .004 & -.028 & -.029 & -.170 & -.108 & .372 \\
\hline
\end{tabular}




\begin{tabular}{|l|r|r|r|r|r|r|r|}
\hline $\mathrm{x} 11$ & .020 & -.005 & .022 & -.087 & -.036 & .473 & -.136 \\
\hline $\mathrm{x} 12$ & -.038 & -.064 & .021 & -.023 & .515 & -.061 & -.038 \\
\hline $\mathrm{x} 13$ & -.011 & -.059 & .004 & -.041 & .491 & -.002 & .000 \\
\hline $\mathrm{x} 14$ & .009 & .380 & .001 & -.071 & -.050 & -.049 & -.056 \\
\hline $\mathrm{x} 15$ & -.005 & .375 & .020 & -.062 & -.039 & -.049 & -.065 \\
\hline $\mathrm{x} 16$ & -.040 & -.190 & -.019 & .064 & .123 & -.017 & .637 \\
\hline $\mathrm{x} 17$ & .207 & -.035 & -.045 & -.045 & .072 & .022 & .006 \\
\hline $\mathrm{x} 18$ & .010 & -.104 & .017 & .407 & -.064 & -.199 & -.087 \\
\hline $\mathrm{x} 19$ & -.008 & .214 & .049 & .174 & -.035 & .118 & -.033 \\
\hline $\mathrm{x} 20$ & -.020 & -.073 & -.032 & .337 & .025 & -.047 & .036 \\
\hline $\mathrm{x} 21$ & -.002 & -.017 & .030 & .350 & -.043 & .175 & -.002 \\
\hline
\end{tabular}

Note: Extraction Method: Principal Component Analysis . Rotation Method :Varimax with Kaiser Normalization.Component Scores.

Table 7 - Component Score Covariance Matrix

\begin{tabular}{|l|r|r|r|r|r|r|r|}
\hline Component & \multicolumn{1}{c|}{1} & \multicolumn{1}{|c|}{2} & \multicolumn{1}{c|}{3} & \multicolumn{1}{l|}{4} & \multicolumn{1}{l|}{5} & \multicolumn{1}{l|}{7} \\
\hline 1 & 1.000 & .000 & .000 & .000 & .000 & .000 & .000 \\
\hline 2 & .000 & 1.000 & .000 & .000 & .000 & .000 & .000 \\
\hline 3 & .000 & .000 & 1.000 & .000 & .000 & .000 & .000 \\
\hline 4 & .000 & .000 & .000 & 1.000 & .000 & .000 & .000 \\
\hline 5 & .000 & .000 & .000 & .000 & 1.000 & .000 & .000 \\
\hline 6 & .000 & .000 & .000 & .000 & .000 & 1.000 & .000 \\
\hline 7 & .000 & .000 & .000 & .000 & .000 & .000 & 1.000 \\
\hline
\end{tabular}

Note: Extraction Method: Principal Component Analysis . Rotation Method :Varimax with Kaiser Normalization.Component Scores.

\section{Acknowledgements}

The authors wish to thank Professor Jianhong Wang for his excellent research assistance, as well as the support by the "Fundamental Research Funds for the Central Universities".

\section{References}

1. Babcock, G. C., When Is Growth Sustainable, Financial Analysts Journal 26(1970), 108-14.

2. Higgins, R. C., How Much Growth Can A Firm Afford, Financial Management 6(1977),7-16

3. Gerard T. Olson, Michael S. Pagano, A New Application of Sustainable Growth: A Multi-Dimensional Framework for Evaluating the Long Run Performance of Bank Mergers, Journal of Business Finance and Accounting 32(2005),1995-2036

4. Higgins, R. C., Analysis for Financial Management, Chinese sixth ed. McGraw-Hill, New York, 88-89

5. Van Home, James C., Sustainable Growth Modelling, Journal of Corporate Finance 1, (1988), 18-32

6. L. H Wang, J. H Han and S. D Gan, Analysis and Evaluation and Reconstruction on Sustainable Growth Model, Statistics and Decision 5(2015),78-80

7. URL:http://cyyw.cena.com.cn/2016-01/19/content_313958.htm(19.1.2016) 